\title{
Protective Effect of Glycine against Liposome Membrane-Perturbation and Lysis Induced by Chlorpromazine
}

\author{
Yasuko Morimoto* and Yoshikazu TaKeuchi \\ Department of Pharmaceutics, Faculty of Pharmaceutical Sciences, Kobe-Gakuin University, Arise, Ikawadani-cho, \\ Nishi-ku, Kobe 651-21, Japan. Received March 3, 1994; accepted May 19, 1994
}

\begin{abstract}
The protective effect of glycine against the perturbing action of chlorpromazine (CPZ) on liposome membranes was investigated by an electron spin resonance (ESR) technique. Analysis using a 5-doxyl stearic acid (DS) spin probe indicated that the hydrophobic region near the surface of the liposomes containing glycine was restricted in motion by the addition of CPZ. This result suggested that the spaces between hydrocarbons in this portion would be narrowed by the penetrance of cationized CPZ molecules into the polar-hydrocarbon interface of the liposomes, in which the surface region may be tightly packed by glycine. The presence of glycine caused no difference in the disordering action of CPZ in the 12-DS reporting portion, suggesting that glycine could not influence the depth of the penetrance of cationized CPZ into the hydrocarbon phase near the surface of liposomes. The perturbing action of CPZ on the 16-DS-labeled liposomes was weakened by the presence of glycine, suggesting the reduced penetrance of undissociated CPZ molecules into the center of the bilayers (lipid core). Thus, glycine presumably prevented CPZ molecules incorporated into the liposome surface from transferring into the lipid core by its packing effect. It was shown that glycine could reduce the liposome lytic action of $\mathrm{CPZ}$, without decreasing its surface activating action. This observed anti-lytic effect could be explained as a result of the above-described inhibition of the penetrance of CPZ into the lipid core.
\end{abstract}

Keywords chlorpromazine; glycine; liposome; electron spin resonance; liposome lysis; lipid core

Amphipathic drugs such as chlorpromazine (CPZ) protect erythrocytes against hypotonic hemolysis, but also elicit a detergent-like hemolysis. ${ }^{1)}$ The hemolytic mechanism has been investigated to elucidate the drug action on biomembranes. Ogiso et al. ${ }^{2 a)}$ reported that the disruptive action of CPZ on the lipid bilayers of erythrocyte membranes possibly induced the hemolysis. Many previous reports have indicated that $\mathrm{CPZ}$ can induce the disturbance or fluidization of lipid bilayers in both biological and artificial membranes. ${ }^{2)}$ In our previous study, ${ }^{3)}$ using an electron spin resonance (ESR) technique, we showed that the addition of CPZ to model lipid membranes (liposomes) consisting of phosphatidylcholine (PC), cholesterol (CHL) and dicetylphosphate (DCP) in a ratio of $1 / 0.75 / 0.1$ induced a marked increase in the mobility of fatty acyl chains at the innermost portion where the 16-doxyl stearate (16-DS) ESR probe was localized, suggesting the penetrance of undissociated CPZ molecules into the center of the bilayers. When the concentration of CPZ was higher than the lipid concentration, liposomal lysis was observed, possibly due to the extensive entrance of CPZ into the inner portion of liposomes. Such an action of CPZ may be associated with the hemolytic mechanism. Lieber et $a l .{ }^{4)}$ reported that a CPZ-rich zone at the center of the bilayers and holes with a diameter of approximately $14 \AA$ were formed on the erythrocyte membranes in the process of CPZ-induced hemolysis. This finding was quite consistent with our proposed mechanism of CPZ action on liposome membranes.

We investigated the protective effects of amino acids on the hemolytic action of CPZ. Previously, we reported that several amino acids retarded both the lag time and the half-life of CPZ-induced hemolysis. ${ }^{5)}$ These amino acids reduced the membrane affinity of $\mathrm{CPZ}$, leading to retardation of hemolysis. ${ }^{5 a)}$ Also, glycine, the simplest amino acid, was found to suppress the perturbing action of CPZ on $\mathrm{PC} / \mathrm{CHL} / \mathrm{DCP}(1 / 0.75 / 0.1)$-liposomes by Fourier transformation infrared (FT-IR) spectroscopy. ${ }^{5 b)}$ In the present study, we attempted to elucidate the mechanism of the inhibiting effect of glycine on the membraneperturbing action of CPZ. For this purpose, we determined the local mobility of ESR probes in liposomes prepared with glycine (Gly-liposomes) in the absence or presence of CPZ; the changes in mobility of these probes in the Gly-liposomes induced by CPZ were compared with the CPZ-induced changes in liposomes hydrated in saline (control liposomes).

\section{Experimental}

Materials $\mathrm{CPZ} \mathrm{HCl}$ was purchased from Wako Pure Chemical Industries, Ltd. Glycine (Gly), DCP and CHL were purchased from Nacalai Tesque, Inc. L- $\alpha$-Phosphatidylcholine (PC) from egg yolk (99\%) was purchased from Sigma Chemical Co. 5-, 12-, and 16-DSs were purchased from Aldrich Chemical $\mathrm{Co}$. These chemicals and other reagents used were of analytical grade.

Liposome Preparation Liposomes were prepared as described previously. ${ }^{3)}$ Two $\mathrm{ml}$ of $\mathrm{PC}(10 \mathrm{mg} / \mathrm{ml})$ containing $\mathrm{CHCl}_{3}$ solution, and $10 \mathrm{ml}$ of $\mathrm{CHCl}_{3}$ solution, in which both DCP $(0.147 \mathrm{mg} / \mathrm{ml})$ and CHL $(0.782 \mathrm{mg} / \mathrm{ml})$ were dissolved, were mixed in a $50 \mathrm{ml}$ round-bottomed flask. Spin probes were dissolved in $\mathrm{CHCl}_{3}$ to a final concentration of $1.25 \mathrm{mg} / \mathrm{ml}$, and the required volume of this solution $(92.3 \mu \mathrm{l})$ was then added to the lipid mixture. The obtained mixture was dried to a thin lipid film on the wall of the round-bottomed flask under reduced pressure in a rotary evaporator. Just before ESR measurement, the film was hydrated in $2 \mathrm{ml}$ of $0.30 \mathrm{M}$ glycine solution and dispersed by vortexing to produce Gly-liposomes. The obtained liposomes contained a spin probe at a concentration of $0.6 \mathrm{~mol} / \mathrm{mol} \%$ of total lipids $(\mathrm{PC} / \mathrm{CHL} /$ $\mathrm{DCP}=1 / 0.75 / 0.1)$. When the liposomes were treated with $\mathrm{CPZ}, 20 \mu \mathrm{l}$ of $0.2 \mathrm{M} \mathrm{CPZ}$ solution containing $\mathrm{NaCl}$ for osmotic equivalence to the $0.15 \mathrm{M}$ $\mathrm{NaCl}$ solution (saline solution), and $20 \mu \mathrm{l}$ of the saline solution were added to $80 \mu \mathrm{l}$ of the prepared liposome suspension. This prepared sample contained $200 \mathrm{~mm}$ glycine and $33.3 \mathrm{~mm} \mathrm{CPZ}$, double the total lipid concentration in the liposome suspension. The desired CPZ concentration in the liposome sample was prepared by changing the volume ratio of $0.2 \mathrm{M} \mathrm{CPZ}$ solution to saline solution. Liposomes hydrated in saline solution were used as controls.

ESR Measurements ESR measurements were carried out as pre- 
viously described. ${ }^{3)}$ A JEOL JES FE-1X spectrometer was used to determine ESR spectra at ambient temperature $\left(21-23^{\circ} \mathrm{C}\right)$, and instrumental parameters were optimized to avoid artificial broadening. The conditions for measurement were as follows: microwave power $20 \mathrm{~mW}$ for 5 -DS or $10 \mathrm{~mW}$ for 12- and 16-DS; microwave frequency, $9.429 \mathrm{GHz}$; magnetic field, $3330 \pm 50 \mathrm{G}$; response, $1 \times 1000$; response time, $0.3 \mathrm{~s}$; modulation, $100 \mathrm{kHz}$; modulation amplitude, $1 \mathrm{G}$ for 5 - and 12-DS or $0.5 \mathrm{G}$ for 16-DS. Order parameters were calculated from hyperfine splitting tensors according to the method described by Hubbell and McConnell, $\left.{ }^{6}\right)$ the parallel and perpendicular components of which, $T^{\prime}$ and $T_{\perp}^{\prime}$, were derived from the spectra as indicated in Fig. la, and the order parameter, $S$, was calculated from the following equations:

$$
\begin{aligned}
& S=\frac{T_{\| l}^{\prime}-T_{\perp}^{\prime}}{T_{\mathrm{zz}}-\left(T_{\mathrm{xx}}+T_{\mathrm{yy}}\right) / 2} \cdot \frac{a_{\mathrm{N}}}{a_{\mathrm{N}}^{\prime}} \\
& a_{\mathrm{N}}^{\prime}=\left(T_{/ /}^{\prime}+2 T_{\perp}^{\prime}\right) / 3 \\
& a_{\mathrm{N}}=\left(T_{\mathrm{xx}}+T_{\mathrm{yy}}+T_{\mathrm{zz}}\right) / 3
\end{aligned}
$$

The values of $a_{\mathrm{N}}$ and $a_{\mathrm{N}}^{\prime}$ are isotropic interactions of nitroxide radicals on solvent polarity. The elements of the hyperfine tensors in the principal directions, $T_{\mathrm{xx}}, T_{\mathrm{yy}}$ and $T_{\mathrm{zz}}$, were taken from Gaffney and McConnell. ${ }^{7 \text { ) }}$ The behavior of 16-DS was analyzed using the parameter of $T_{1 / .}^{\prime 3,8)}$

Critical Micelle Concentration (cmc) Determination The $\mathrm{cmc}$ value for $\mathrm{CPZ}$ in the solution of $200 \mathrm{~mm}$ glycine, which was adjusted to be osmotically equivalent to the saline solution by adding $\mathrm{NaCl}$, was determined according to the fluorescence method of Luxnat and Galla, ${ }^{9}$ as previously used to determine the cmc of CPZ in saline solution. ${ }^{3)}$ This method is based on the supposition that aggregates of CPZ molecules are spectroscopically different from the monomer in solution. The fluorescence spectra of CPZ in $200 \mathrm{~mm}$ glycine solution were taken as a function of $\mathrm{CPZ}$ concentrations with a fluorescence spectrometer (Hitachi fluorescence spectrophotometer F-3000). Excitation spectra were measured at a fixed emission wavelength of $450 \mathrm{~nm}$. From the obtained data, the $\mathrm{cmc}$ value was calculated as the starting point at which a step-like increase in the excitation maximum occurred, and the obtained value was compared with the $\mathrm{cmc}$ of $\mathrm{CPZ}$ in saline.

Lysate Determination Both Gly- and control liposomes were prepared as described above, except that the spin probe was not included. To induce lysis, both species of liposomes were treated with $\mathrm{CPZ}$ at a ratio of $\mathrm{CPZ} / \mathrm{lipid}=2$, i.e. $100 \mu \mathrm{l}$ of $\mathrm{CPZ}$ solution $(0.2 \mathrm{M})$ and $100 \mu \mathrm{l}$ of saline solution were added to $400 \mu \mathrm{l}$ of the liposome suspension. The mixtures were gently shaken for $20 \mathrm{~min}$ in a Taiyo incubator M-100N at ambient temperature, then centrifuged for $15 \mathrm{~min}$ at $14000 \mathrm{rpm}$ in a Kubota KR-20000S. The concentration of phospholipid in the supernatant was determined using a Phospholipid-test kit (Wako Pure Chemical Industries, Ltd.).

\section{Results and Discussion}

Determination of ESR Parameters ESR spectra of 5-, 12- or $16-\mathrm{DS}$ in $\mathrm{PC} / \mathrm{CHL} / \mathrm{DCP}$ (1/0.75/0.1)-liposomes, which were hydrated in either $0.3 \mathrm{M}$ glycine solution (Gly-liposomes) or the saline (control liposomes), and were treated with or without $\mathrm{CPZ}$ at $22^{\circ} \mathrm{C}$ are shown in Figs. $1-3$. From these spectra, order parameters for 5- or 12-DS in liposome membranes were calculated with each sample to evaluate the degree of membrane ordering or disordering at the sites where these two probes were localized. The line shape of the ESR spectra for 16-DS probes, which report the molecular mobility of hydrophobic chains in the innermost portion, in the Gly-liposomes was changed by treatment of liposomes with CPZ. The Gly-liposomes not treated with $\mathrm{CPZ}$ produced an anisotropic spectrum, which had a shoulder on the peak at the lower magnetic field and two peaks at the higher magnetic field (Fig. 3c), while those with CPZ-treatment gave an isotropic spectrum without either the shoulder or the distinction of two peaks (Fig. 3d). Such a change induced by CPZ in the spectral shape of this probe was also observed in the

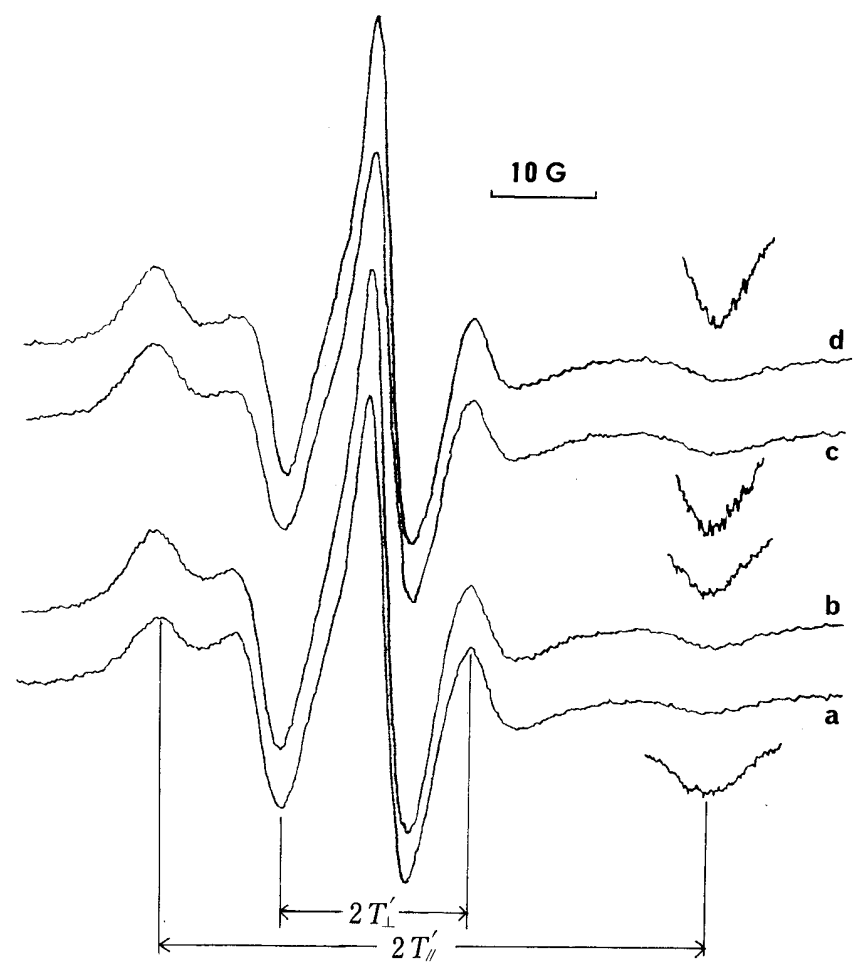

Fig. 1. ESR Spectra of 5 -DS in the PC/CHL/DCP $(1: 0.75: 0.1)$ Liposomes

$\mathrm{a}, \mathrm{b}$, hydrated in saline; $\mathrm{c}, \mathrm{d}$, hydrated in $0.3 \mathrm{M}$ glycine solution, and $\mathrm{a}, \mathrm{c}$, untreated with $\mathrm{CPZ}$; b, d, treated with $\mathrm{CPZ}$ at $\mathrm{CPZ} / \mathrm{lipid}$ ratio $=0.4$.

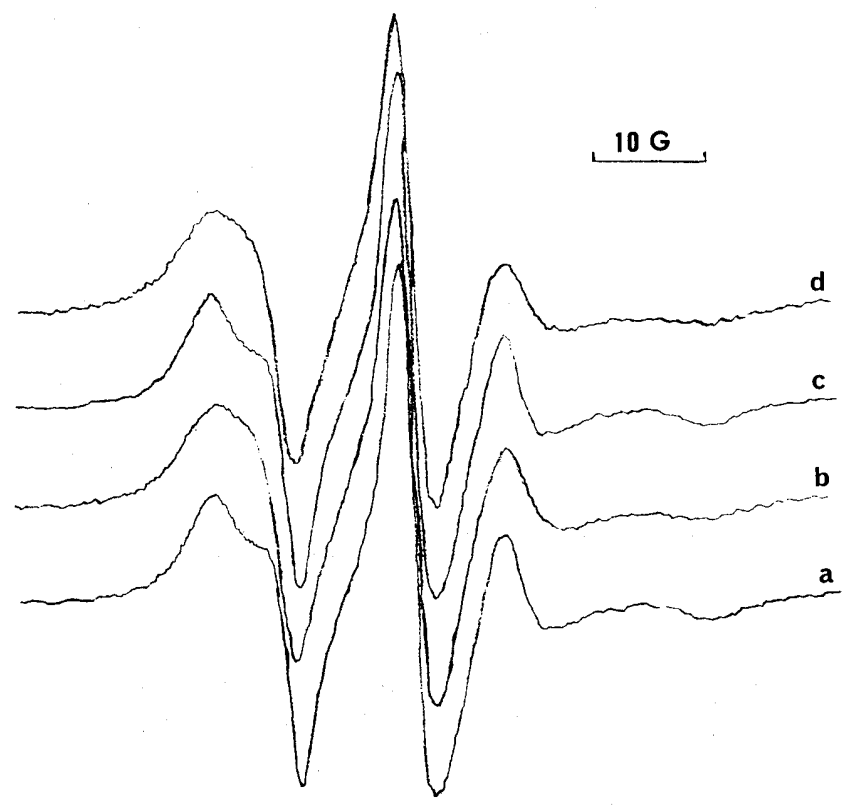

Fig. 2. ESR Spectra of $12-\mathrm{DS}$ in the $\mathrm{PC} / \mathrm{CHL} / \mathrm{DCP}(1: 0.75: 0.1)$ Liposomes

$\mathrm{a}, \mathrm{b}$, hydrated in saline; $\mathrm{c}, \mathrm{d}$, hydrated in $0.3 \mathrm{M}$ glycine solution, and $\mathrm{a}, \mathrm{c}$, untreated with $\mathrm{CPZ} ; \mathrm{b}, \mathrm{d}$, treated with $\mathrm{CPZ}$ at $\mathrm{CPZ} /$ lipid ratio $=0.4$.

control liposomes (Figs. 3a, 3b). With such spectral shape transition, neither the order parameter nor the rotational correlation times were appropriate for evaluation of the molecular motion of 16-DS in the liposomes, and a hyperfine splitting tensor, $T_{/ /}^{\prime}$, was instead introduced to evaluate the effect of $\mathrm{CPZ}$ on this molecular motion. As 


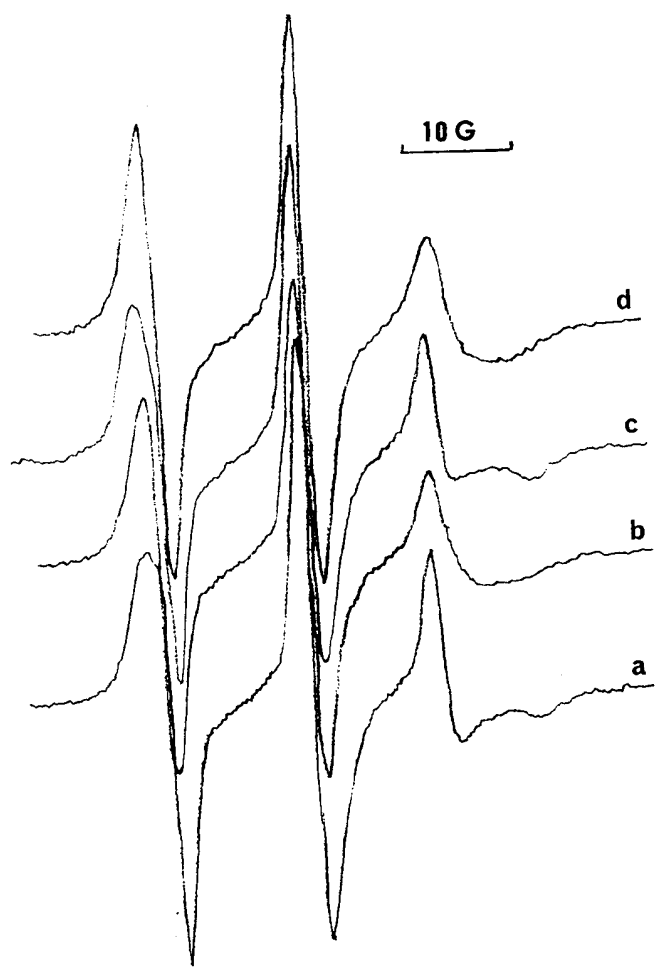

Fig. 3. ESR Spectra of $16-\mathrm{DS}$ in the PC/CHL/DCP $(1: 0.75: 0.1)$ Liposomes

$\mathrm{a}, \mathrm{b}$, hydrated in saline; $\mathrm{c}, \mathrm{d}$, hydrated in $0.3 \mathrm{~m}$ glycine solution, and $\mathrm{a}, \mathrm{c}$, untreated with $\mathrm{CPZ}$; b, d, treated with $\mathrm{CPZ}$ at $\mathrm{CPZ} /$ lipid ratio $=0.4$.

TABLE I. Effects of Glycine on the Changes Induced by CPZ in Order Parameter for 5-DS in Liposomes

\begin{tabular}{ccc}
\hline \hline \multirow{2}{*}{$\begin{array}{c}\text { CPZ/lipid } \\
\text { ratio }\end{array}$} & \multicolumn{2}{c}{ Order parameter } \\
\cline { 2 - 3 } & Control & Gly-liposome \\
\hline 0 & $0.639 \pm 0.003$ & $0.651 \pm 0.008^{a)}$ \\
0.4 & $0.640 \pm 0.007$ & $0.670 \pm 0.003^{c}$ \\
0.6 & $0.640 \pm 0.011$ & $0.670 \pm 0.003^{a)}$ \\
1.0 & $0.641 \pm 0.008$ & $0.671 \pm 0.005^{c)}$ \\
2.0 & $0.604 \pm 0.009$ & $0.625 \pm 0.010^{b)}$ \\
3.0 & $0.569 \pm 0.010$ & $0.594 \pm 0.016^{a)}$ \\
4.0 & $0.536 \pm 0.017$ & $0.568 \pm 0.004^{a)}$ \\
\hline
\end{tabular}

Each order parameter value represents the mean + S.D. of three to eight values. Significantly different from the values for the control liposomes: a) $p<0.05$ b) $p<0.01 ; c) p<0.005$.

previously reported, ${ }^{3)}$ the changes in $T_{\|}^{\prime}$ values with $\mathrm{CPZ} /$ lipid ratio for 5-DS or 12-DS in control liposomes paralleled those in the order parameters, and the $T_{/ /}^{\prime}$ value was found to be capable of reporting the molecular mobility of hydrocarbons at the site where the nitroxide moiety of the probe was localized. The calculated parameters were plotted against the molar ratio of $\mathrm{CPZ}$ in the liposome suspension. The results are shown in Fig. 4 , and the values are summarized in Tables I-III.

Effects of Glycine on the Packing of CPZ-Free Liposomes The order parameter values for the 5-DS probe in the Gly-liposomes were significantly higher than those in the controls (Table I), suggesting that the hydrophobic chains near the surface were tightly packed by glycine. The molecular motion of $12-\mathrm{DS}$ and $16-\mathrm{DS}$
TABLE II. Effects of Glycine on the Changes Induced by CPZ in Order Parameter for 12-DS in Liposomes

\begin{tabular}{ccc}
\hline \hline \multirow{2}{*}{$\begin{array}{c}\text { CPZ/lipid } \\
\text { ratio }\end{array}$} & \multicolumn{2}{c}{ Order parameter } \\
\cline { 2 - 3 } & Control & Gly-liposome \\
\hline 0 & $0.521 \pm 0.005$ & $0.514 \pm 0.008$ \\
0.4 & $0.478 \pm 0.013$ & $0.486 \pm 0.007$ \\
0.6 & $0.493 \pm 0.007$ & $0.493 \pm 0.001$ \\
1.0 & $0.515 \pm 0.013$ & $0.517 \pm 0.009$ \\
2.0 & $0.514 \pm 0.007$ & $0.527 \pm 0.008$ \\
3.0 & $0.518 \pm 0.008$ & $0.512 \pm 0.013$ \\
4.0 & $0.527 \pm 0.007$ & $0.516 \pm 0.014$ \\
\hline
\end{tabular}

Each order parameter value represents the mean \pm S.D. of three to five values. The values for the Gly-liposomes were not significantly different from those for the control liposomes at any $\mathrm{CPZ} /$ lipid ratio.

TABLE III. Effects of Glycine on the Changes Induced by CPZ in Hyperfine Splitting for 16-DS in Liposomes

\begin{tabular}{ccc}
\hline \hline \multirow{2}{*}{$\begin{array}{c}\text { CPZ/lipid } \\
\text { ratio }\end{array}$} & \multicolumn{2}{c}{ Order parameter } \\
\cline { 2 - 3 } & Control & Gly-liposome \\
\hline 0 & $17.59 \pm 0.28$ & $17.75 \pm 0.33$ \\
0.2 & $15.84 \pm 0.25$ & $16.39 \pm 0.22^{b)}$ \\
0.4 & $15.47 \pm 0.18$ & $15.85 \pm 0.13^{a)}$ \\
0.6 & $15.65 \pm 0.25$ & $15.71 \pm 0.29$ \\
0.8 & $15.52 \pm 0.17$ & $15.83 \pm 0.40$ \\
1.0 & $15.81 \pm 0.21$ & $15.86 \pm 0.37$ \\
2.0 & $15.74 \pm 0.14$ & $15.39 \pm 0.17$ \\
3.0 & $16.05 \pm 0.31$ & $16.04 \pm 0.27$ \\
4.0 & $16.05 \pm 0.17$ & $16.12 \pm 0.31$ \\
\hline
\end{tabular}

Each order parameter value represents the mean \pm S.D. of three to seven values. Significantly different from the values for the control liposomes: $a$ ) $p<0.05$; b) $p<0.01$.

probes, localized to the middle and innermost portions of fatty acyl chains, respectively, in the Gly-liposomes were similar to those in the control liposomes (Tables II, III); thus no packing effect of glycine was observed at the inner hydrophobic region of the liposomes. The restriction of the 5-DS probe observed in the Gly-liposomes may be due to the interaction of glycine with polar head groups. Previously, we observed that the fatty acyl chains near the surface of liposomes were more restricted in motion at $\mathrm{pH}$ 6.4 than at $\mathrm{pH} 6.0$, and that the molecular mobility of the inner hydrophobic region was not influenced by differences in $\mathrm{pH}$ value of the suspension. ${ }^{10)}$ The $\mathrm{pH}$ value of the glycine solution used in the present study was 6.2 , higher than the value of 5.7 for the saline which was used to suspend the control liposomes. Based on these findings, it is also possible to presume that the restriction of the 5-DS probe in the Gly-liposomes described above was caused by the higher $\mathrm{pH}$ of the glycine solution than the saline. Further study is necessary to elucidate the interaction between glycine and the polar surface of liposomes.

Protective Effects of Glycine on the MembranePerturbing Action of CPZ When CPZ was added to the 5-DS-labeled liposome suspension up to an equimolar CPZ to lipid ratio $(\mathrm{CPZ} / \mathrm{lipid} \leqq 1)$, the order parameter values of the probe in the control liposomes were hardly changed, while those in the Gly-liposomes were significantly 

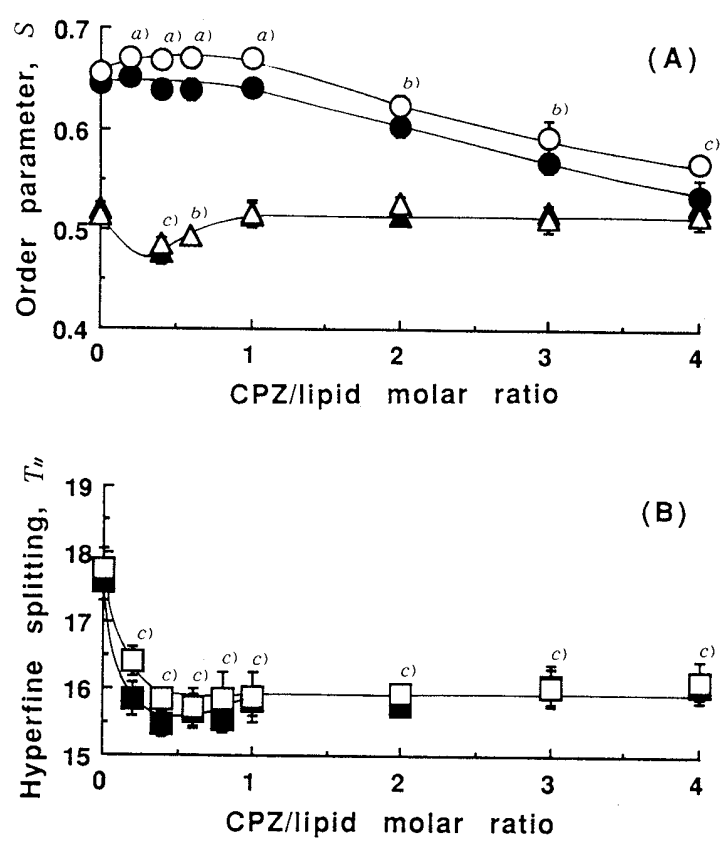

Fig. 4. Effects of CPZ on (A) Order Parameters for 5-DS $(0,0)$ and 12-DS $(\mathbf{\Delta}, \triangle)$; (B) Hyperfine Splitting for 16-DS $(\square, \square)$, in the Liposomes at $22^{\circ} \mathrm{C}$

Closed symbols, control liposomes; open symbols, Gly-liposomes. Significantly different from the value for Gly-liposomes untreated with CPZ: $a$ ) $p<0.05 ; b)$ $p<0.01 ; c) p<0.001$.

increased (Fig. 4A,$O$ ). Such an ordering effect of CPZ on the hydrophobic chains near the surface of the Gly-liposomes may be a result of the penetrance of cationized CPZ molecules into the polar-hydrocarbon interface. The aromatic moiety of the $\mathrm{CPZ}$ molecules may enter the spaces between hydrocarbon chains near the surface of liposomes where the 5-DS is localized, inducing a narrowing of these spaces and leading to the observed restriction in molecular mobility of the 5-DS probe. Such narrowed spaces may possibly be caused by the packing effect of glycine on the surface polar region of liposomes as described above. On the other hand, the surface region of the control liposomes was packed more loosely than that of the Gly-liposomes, and the cationized CPZ molecules in the polar-hydrocarbon interface could probably cause an expansion of the spaces between the hydrophobic chains near the surface and so that they would be incorporated as a liposomal component.

By addition of CPZ at a CPZ/lipid ratio $>1$, the molecular mobility of the 5-DS probe in the Glyliposomes was enhanced as the CPZ/lipid ratio was increased. Such enhancement in the mobility of this probe was also observed in the control liposomes treated with $\mathrm{CPZ}$ at a $\mathrm{CPZ} /$ lipid ratio $>1$. In our previous report, ${ }^{3)}$ this phenomenon was explained as a consequence of liposomal lysis leading to the formation of mixed micelles consisting of lipids and CPZ, in which the nitroxide group of the 5-DS probe would be loosely packed. The observed enhancement in the mobility of the 5-DS in the Glyliposomes may also be due to the occurrence of liposomal lysis induced by CPZ. As shown in Table I, whenever either type of liposome was treated with CPZ at any $\mathrm{CPZ} /$ lipid ratio $>1$, the values of the 5 -DS order pa- rameter in the Gly-liposomes were significantly larger than those in the controls. This suggests that the hydrophobic region near the surface of the Gly-liposomes may be more rigid than that in controls under the CPZ-loaded conditions. Also, the lysate of the Gly-liposomes may be produced in smaller quantities than that of the control liposomes on treatment with the same concentrations of CPZ. To investigate this possibility, phospholipid leakage into the supernatant was examined with both types of liposomes treated with double concentrations of CPZ to lipid. Phospholipids in the supernatant of Gly-liposomes were quantified as $3.309 \pm 0.216(\mu \mathrm{g})$, significantly less than in that from control liposomes, $3.691 \pm 0.227(\mu \mathrm{g})$ $(p<0.05)$, and the \% of lysates was estimated as $85.5 \%$ and $96.6 \%$, respectively. This result demonstrated that the Gly-liposomes were less easily lysed by treatment with $\mathrm{CPZ}$ than the control liposomes. At higher pHs, CPZ induced disorder in the 5-DS probe in liposomes to a greater degree, suggesting an increase in severity of liposomal lysis. ${ }^{10)}$ The $\mathrm{pH}$ values of glycine solution containing CPZ at concentrations corresponding to $\mathrm{CPZ} /$ lipid ratios of 1 to 4 were 6.0 to 5.6, respectively, and each value was higher than the $\mathrm{pH}$ of the saline, 5.3-4.9, containing CPZ at the same concentration. On the basis of these findings, the above observation indicating that the Gly-liposomes were less easily lysed than the control liposomes may be explained as a result of the protective effect of glycine exceeding the enhancing effect of the higher $\mathrm{pH}$ on the liposomal lysis induced by CPZ.

As we reported previously, ${ }^{3)}$ the lysis of liposomes possibly occurred with excessive penetration of CPZ molecules into the lipid membranes. Thus, the decrease in the lytic action of CPZ observed in the Gly-liposomes may be a consequence of the inhibiting effect of glycine on the penetrance of CPZ into the liposomes. However, Luxnat and $\mathrm{Galla}^{9}$ reported that liposomal lysis was induced by the addition of CPZ at concentrations higher than the cmc when the lipid concentration was very low. Their study suggested that the lysis of liposomes could be induced by the surface activating action of CPZ. If glycine reduced this surface activating action, the collapse of the Glyliposomes could be reduced. To investigate this possibility, the $\mathrm{cmc}$ value of $\mathrm{CPZ}$, which was regarded as a good index of the surface activating action, was measured in glycine solution, and compared with the value in saline. The $\mathrm{cmc}$ values in both glycine and saline solutions were measured by fluorometry as described in the Experimental section. The excitation maximum, $\lambda_{\max }$, for each spectrum of CPZ at various concentrations in each solution was measured. The wavelength, $\lambda_{\max }$, was plotted as a function of CPZ concentration, and the results are shown in Fig. 5. The change in $\lambda_{\max }$ value with increasing CPZ concentration in the glycine solution $(O)$ was in good agreement with that in the saline solution (O). In either case, a step-like increase in $\lambda_{\max }$ value was observed at a CPZ concentration of $0.3 \mathrm{~mm}$. Thus, the cmc value of CPZ in both glycine and saline solutions was estimated as $0.3 \mathrm{~mm}$. On the basis of this finding, the assumption described above was unfounded, i.e. glycine could not reduce the surface activating action of CPZ.

The disordering action of $\mathrm{CPZ}$ observed in the middle 


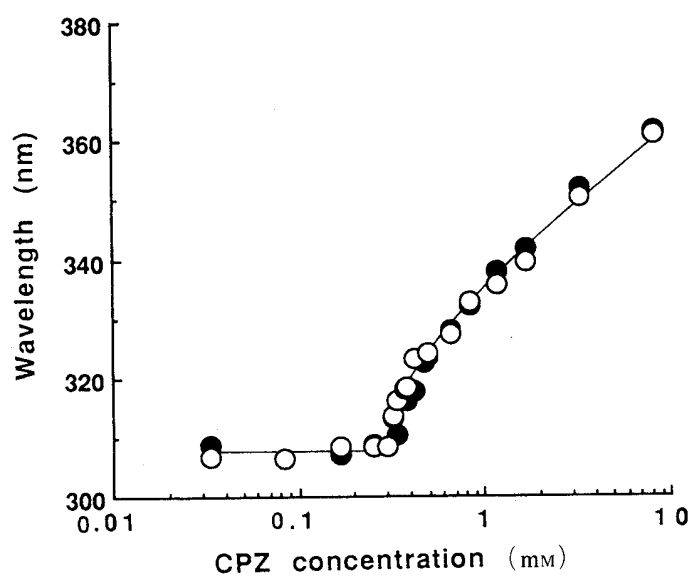

Fig. 5. Excitation Maximum of Spectra Shown for CPZ Solution, in the Presence $(O)$ or Absence (O) of $200 \mathrm{~mm}$ Glycine, as a Function of CPZ Concentration

The fluorescence was measured at $450 \mathrm{~nm}$.

portion of fatty acyl chains, where 12-DS was localized, in the Gly-liposomes was maximal at a CPZ/lipid ratio $=0.4$, and was almost the same as that in the controls (Fig. 4A $\triangle, \Delta$ ). As we reported previously, ${ }^{3)}$ the disordering action of CPZ in this portion of the control liposomes could be explained as a result of the penetrance of cationized CPZ into the polar-hydrocarbon interface leading to the expansion of the spaces between hydrocarbon chains at the 12 -DS reporting area. The disordering action of CPZ observed in the 12-DS-labeled Gly-liposomes may also be explained as a result of the penetrance of CPZ into the polar-hydrocarbon interface. The aromatic moiety of CPZ molecules inserted into the interface, which probably narrowed the spaces between the hydrocarbon chains near the 5-DS reporting portion as described above, may induce the expansion of the spaces in the inner portion, where the 12-DS was localized. Previously, we explained the disappearance of the disordering action of $\mathrm{CPZ}$ at higher concentration in control liposomes as a consequence of the cationized $\mathrm{CPZ}$ molecules which had been incorporated into the surface region, transferring to the inner portion during the molecular rearrangement of the lipids and CPZ, and presumably filling up the space compartment. ${ }^{3)}$ In the Glyliposomes, the disappearance of the disordering action of CPZ may be due to such a rearrangement of lipids and CPZ. As shown in Table II, there were no significant differences in the observed values of order parameters for 12-DS between the Gly- and the control liposomes at any CPZ/lipid ratio from 0.2 to 4 . This suggests that glycine could not influence the depth of the penetrance of cationized $\mathrm{CPZ}$ molecules into the hydrocarbon phase near the surface of the liposomes.

As shown in Fig. 4B, the $T_{/ /}$values for the Gly-liposomes labeled with the 16-DS probe were markedly reduced, as were those of the control liposomes. The 16-DS spectrum in the Gly-liposomes not treated with CPZ had two components (Fig. 3c), while the spectrum in those treated with CPZ had only one component, with smaller $T_{/ /}$values (Fig. 3d). Such a spectral shape change attended by the decrease in $T_{/ /}$values was also observed in the control liposomes (Figs. 3a, 3b), and was explained in our previous report $^{3)}$ as follows. The two components of the spectrum were likely attributable to the different locations of the nitroxide group of the 16-DS probe in the bilayers, one at the center (lipid core) and the other in the hydrocarbon phase where the label must be more restricted in motion. The location of the label in the hydrocarbon phase was possibly a result of the great amplitude of flexing motion of the almost undissociated probe about the axis perpendicular to the bilayer. ${ }^{11)}$ In the presence of $\mathrm{CPZ}$, most of the label may exist in the lipid core, which was presumably widened by the entrance of the undissociated CPZ molecules, and this phenomenon would exhibit a single spectral component with smaller $T_{/ /}$value. These explanations may be appropriate for the current observation with Gly-liposomes. When the spin probe was partially ionized, two forms of which would be anchored at different positions in the polar interface, it may have exhibited two spectral components. At the $\mathrm{pH}$ of the $200 \mathrm{~mm}$ of glycine solution (6.2), which was used to suspend the Gly-liposomes in the present study, about $80 \%$ of the probe molecules were expected to be undissociated. ${ }^{2)}$ Thus, the appearance of the two components in the 16-DS spectrum in the Gly-liposomes (Fig. 3c) could not be explained by the partial ionization of the probe, and was regarded possibly as a result of the perpendicular flexing of the probe molecules relative to the bilayer. Even in the presence of CPZ in the $200 \mathrm{~mm}$ glycine solution at a CPZ/lipid ratio of 0.2 or 4 , and at a $\mathrm{pH}$ of 6.2 or 5.6 , respectively, the almost undissociated probes would be flexing perpendicularly to the bilayers. In this case, the lipid core of the Gly-liposomes was presumably widened by the incorporation of undissociated CPZ molecules, as true in the control liposomes.

As shown in Table III, the observed values of $T_{\|}$for the 16-DS probe in the Gly-liposomes were significantly larger than those in the control liposomes, when they were treated with $\mathrm{CPZ}$ at a CPZ/lipid ratio of 0.2 or 0.4 . The reduction in $T_{/ /}$values induced by $\mathrm{CPZ}$ at $\mathrm{pH} 6.4 \mathrm{did}$ not significantly differ from that at $\mathrm{pH} 6.0 .^{10}$ ) Thus, the observed decrease in the disorderring action of $\mathrm{CPZ}$ in Gly-liposomes could be explained as a result of the inhibiting effect of glycine on the penetrance of undissociated CPZ into the center of the bilayers. As described above, the penetrance of cationized CPZ into the hydrophobic region near the surface of liposomes was not influenced by glycine. From these results, glycine was capable of preventing the CPZ molecules which had entered into the hydrophobic phase near the surface of the liposomes from transferring into the center of the bilayers. And such a decrease in the penetrance of CPZ into the center of the bilayers would result in the diminishing of the lytic action of CPZ as described above. The preventative effect of glycine on the transference of CPZ may be a result of the tight packing of the hydrophobic region near the surface of the Gly-liposomes as shown by analysis using the 5-DS probe. The present study did not provide the experimental data necessary to determine the cause of the packing effect of glycine. The interaction of glycine with the polar head groups of the liposomal bilayers could possibly be the cause of this effect, 
and will be investigated in future.

\section{Conclusion}

The mechanism of the inhibiting action of glycine on membrane-perturbation and the lysis of liposomes induced by CPZ was studied using an ESR technique. Analysis using a 5-DS spin probe showed that the hydrophobic region near the surface of the Gly-liposomes was restricted in motion by addition of CPZ, suggesting that the spaces between hydrocarbons in this portion would be narrowed by the penetrance of cationized CPZ molecules into the polar-hydrocarbon interface. Such narrowed spaces might be caused by the packing effect of glycine on the surface region of the liposomes. It was also shown that the liposomal lysis induced by addition of CPZ at a CPZ/lipid ratio $>1$, occurred less easily in the Gly-liposomes than in the controls. The 12-DS reporting portion of the Gly-liposomes was disordered by CPZ in the same manner as in the control liposomes, suggesting that glycine could not influence the penetrance of cationized CPZ into the polar-hydrocarbon interfaces. In the innermost portion of the hydrophobic chains where the 16-DS probe was localized, the observed disordering action of CPZ in the Gly-liposomes was to a lesser degree than that in the control liposomes. This result showed that the penetrance of undissociated CPZ molecules into the center of the bilayers was probably prevented by the effects of glycine, and such an effect of glycine might have decreased the lytic action of CPZ.

References

1) a) P. Seeman, J. Weinstain, Biochem. Pharmacol., 15, 1737 (1966); b) Idem, ibid., 15, 1753 (1966)

2) a) T. Ogiso, M. Kurobe, H. Masuda, Y. Kato, Chem. Pharm. Bull., 25, 1078 (1977); b) Y. Kanaho, T. Sato, T. Fujii, Mol. Pharmacol., 20, 704 (1981); S. Noji, T. Takahashi, H. Kon, Biochem. Pharmacol., 31, 3173 (1982); T. Suda, D. Shimizu, N. Maeda, T. Shiga, ibid., 30, 2057 (1981); S. K. Han, N. H. Kim, Y. S. Lee, Arch. Pharm. Res., 9, 75 (1986); R. Hanpft, K. Mohr, Biochim. Biophys. Acta, 814, 156 (1985).

3) Y. Morimoto, M. Hosokawa, H. Sayo, Y. Takeuchi, Chem. Pharm. Bull., 42, 123 (1994).

4) M. R. Lieber, Y. Lange, R. S. Weinstain, T. L. Stech, J. Biol. Chem., 259, 9225 (1984).

5) a) Y. Morimoto, K. Kotani, M. Watanabe, Y. Fukumori, T. Fukuda, Y. Takeuchi, Yakuzaigaku, 49, 215 (1989); b) Y. Takeuchi, Y. Yamaoka, Y. Morimoto, I. Kaneko, Y. Fukumori, T. Fukuda, J. Pharm. Sci., 78, 3 (1989).

6) a) W. L. Hubbell, H. M. McConnell, J. Am. Chem. Soc., 93, 314 (1971); b) M. Kouno, T. Shiga, K. Mukai, "Jitsuyo ESR Nyuumon," ed. by K. Ishizu, Kodansha Scientific, Tokyo, 1981, pp. $138-178$.

7) B. J. Gaffney, H. M. McConnell, J. Mag. Res., 16, 1 (1974).

8) N. Muranushi, N. Takagi, S. Muranishi, H. Sezaki, Chem. Phys. Lipids, 28, 269 (1981).

9) M. Luxnat, H. J. Galla, Biochim. Biophys. Acta, 856, 274 (1986)

10) Y. Morimoto, Y. Fukumori, T. Fukuda, Y. Takeuchi, Yakuzaigaku, 52, 231 (1992)

11) J. M. Boggs, B. Tummler, Biochim. Biophys. Acta, 1145, 42 (1993).

12) M. Edret-Charlier, A. Sanson, M. Ptak, FEBS Lett., 89, 313 (1978); A. Sanson, M. Ptak, Chem. Phys. Lipids, 17, 435 (1976). 